\title{
Analyzing on the Decomposition based Pricing Procedure for Solving Two Person Zero Sum Game Problems through Computer Algebra
}

\author{
H. K. Das \\ Dept. of Mathematics University of Dhaka \\ Dhaka 1000, Bangladesh
}

\author{
Sajal Chakroborty \\ Department of Electronics and Communications \\ Engineering \\ East West University, Dhaka 1212, Bangladesh
}

\begin{abstract}
Objective of this paper is to analyze on the decomposition based pricing (DBP) method for solving two person zero sum game problems. Decomposition based algorithms have been developed which is able to solve two person zero sum game problems with single payoff elements using the linear programming (LP). To develop this procedure, idea of DBP method have used. Its computer oriented program has also introduced by using a mathematical programming language (AMPL). A real life oriented problem has introduced to show the efficiency of our algorithm and its program. The ability of our program has shown in saving labor and time for solving game problems by analyzing a number of numerical examples.
\end{abstract}

\section{Keywords}

Game, Pure and Mixed strategy, DBP, LP, Computer Algebra.

\section{INTRODUCTION}

Von Neumann and Morgenstern have developed a very fruitful theory of two person zero sum games in their book [9] "Theory of Games and Economic Behavior". This book also contains a theory of n-person games of a type which is called cooperative. This theory is called matrix games, are interesting in many ways and their analysis is tractable due to their simplicity. Note that when a player tries to maximize his payoff, he is also simultaneously minimizing payoff of the other player. For this reason, these games are also called strictly competitive games. Player 1 is usually called the row player and player 2 is called the column player. Game theory bears a strong relationship to LP, in the sense that two person zero sum game can be expressed as a LP and vice versa. Von Neumann and Morgenstern showed that linear programming can be used to solve these games.

In 1963, G. Dantzig states that when J. von Neumann, father of game theory, when first introduced to the simplex method in 1947, immediately recognized this relationship. This paper is studied on the fundamental concepts about a two-person non-cooperative normal game and then improve a decomposition algorithm and develop its computer oriented program which is able to solve two person zero sum game problems with single payoff elements using LP. In this game, there are two players who do not cooperate and their decision rules and evaluation functions are represented by matrices.

Decomposition based pricing is a very useful solution procedure of optimization problems and widely applicable for solving large scale optimization problems $[2,5,7,13]$. This procedure iteratively solves a relaxed sub-problem to identify potential entering basic columns. The sub-problem is chosen to exploit special structure, rendering it is easy to solve [14]. In this paper, a new approach has been presented to solve problems of game theory by converting it into LP problems. Idea of DBP is used to solve this.

Rest of the paper is organized as follows. Section 2, briefly discuss on the formulation of LP for solving two person zero sum game problems and decomposition based pricing method[12, 16, 17]. The section 3, describe an improved decomposition algorithm for solving two person zero sum game problems. Respectively in Section 4 and 5 are based on the numerical illustrations and computational experiments. Section 6 is presented for the convergence comparison in the introduce algorithm.

\section{PRELIMINARIES}

In the current Section, some relevant discussions for solving two person zero sum game problems have been discussed.

\subsection{Formulation of Game into LP}

This Section illustrates the solution of games by linear programming (LP). Any two person zero sum game with mixed strategies can be solved by transforming the problem to a LP. Let, the value of game is $v$. Initially, player I acts as maximize and player II acts as minimize. But after transforming some steps when LPhave to convert then inverse the value of the game. For this objective function also changes.

Cosider the optimal mixed strategy for player II. Expected payoff for player II be $\sum_{i=1}^{m} \sum_{j=1}^{n} p_{i j} x_{i} y_{j}$ and the player II strategy $\left(y_{1}, y_{2}, \ldots, y_{n-1}, y_{n}\right) \quad$ is optimal if $\sum_{i=1}^{m} \sum_{j=1}^{n} p_{i j} x_{i} y_{j} \leq v \quad$ for $\quad$ all opposing strategies $\left(x_{1}, x_{2}, \ldots, x_{m-1}, x_{m}\right)$. Finally, the followng forms for the player II and player I respectively are found. The readers can find the detail discussion in Section 5.1 of the reference [3]. It has been briefly discussed as follows.

Player II :

Maximize: $\frac{1}{v}=y_{1}+y_{2}+\ldots+y_{n-1}+y_{n}$ 
subject to,

$$
\begin{aligned}
& p_{11} y_{1}+p_{12} y_{2}+\ldots+p_{1 n} y_{n} \leq 1 \\
& p_{21} y_{1}+p_{22} y_{2}+\ldots+p_{2 n} y_{n} \leq 1
\end{aligned}
$$

$p_{m 1} y_{1}+p_{m 2} y_{2}+\ldots+p_{m n} y_{n} \leq 1$

$y_{1}+y_{2}+y_{3}+y_{4}+\ldots+y_{n}=1$

and $y_{j} \geq 0, j=1, \ldots, n$

Player I:

Minimize: $\frac{1}{v}=x_{1}+x_{2}+\ldots+x_{m-1}+x_{m}$

subject to

$p_{11} x_{1}+p_{21} x_{2}+\ldots+p_{m 1} x_{m} \geq 1$

$p_{12} x_{1}+p_{22} x_{2}+\ldots+p_{m 2} x_{m} \geq 1$

$p_{1 n} x_{1}+p_{2 n} x_{2}+\ldots+p_{m n} x_{m} \geq 1$

$x_{1}+x_{2}+x_{3}+x_{4}+\ldots+x_{m}=1$

and $x_{i} \geq 0, i=1, \ldots, m$

Now (2a) and (2b) have to solve to find the strategy of both players.

\subsection{Decomposition Based Pricing Method}

This technique was developed by Mamer and McBride [12]. In this section, decomposition based pricing algorithm briefly has been discussed in the following steps.

Step 1: Relax complicating constraints by subtracting from objective function of the original problem. Decompose the whole problem into sub-problems and a master problem. Solve sub-problems and generate muster problem by deleting those variables which do not provide non negative values from the original problem.

Step 2: Stop when sub problem value and master problem value become equal. Otherwise repeat the previous steps.

\section{ALGORITHM}

This Section improves a decompositin algorithm for the solution of two person zero sum games using DBP method.

Step 1: Search the minimum element from each row of the payoff matrix and then find the maximum element of these minimum elements.

Step 2: Search the maximum element from each column of the payoff matrix and then find the minimum element of these maximum elements

Step 3: For the player I if the Maximin less than zero then find $\mathrm{k}$ which is equal to addition of one and absolute value of Maximin.
Step 4: For the player II if the Minimax less than zero then find $\mathrm{k}$ which is equal to addition of one and absolute value of Minimax.

Step 5: If Maximin and Minimax both are greater than zero then $\mathrm{k}=0$.

Step 6: To construct the modified payoff matrix adding k with each payoff elements of the given payoff matrix.

Step 7: Then to find the mixed strategies with game value of the two players, formulate the game problems in LP like equation (2a) and (2b) of Sub-section 2.1. Then follow the following Sub-steps.

Step 8: Subtract complicating constraint from objective function and generate sub- problems.

Sub-step 1: Solve sub-problem and determine the nonnegative variables.

Sub-step 2: Delete all those variables which are not nonnegative and generate the master problem.

Sub-step 3: Solve master problem.

Sub-step 4: If sub-problem value and master problem value become equal then stop the iterations. Otherwise repeat Sub-steps 1 to 3.

\section{NUMERICAL ILLUSTRATIONS}

In this Section, our algorithm prescribed in Section 3 has been illustrated by considering a number of numerical examples.

\subsection{Numerical example 1}

Find the optimum strategies for two players and the value of the game [3].

$$
\begin{array}{ccc}
y_{1} & y_{2} & y_{3} \\
I & H & H I
\end{array}
$$

Player I $\begin{array}{cc}x_{1} & I \\ x_{2} & I I \\ x_{3} & I I I\end{array}\left[\begin{array}{ccc}1 & 1 & 4 \\ 4 & -2 & 4 \\ -1 & 2 & -2\end{array}\right]$

\section{Solution using our algorithm}

Let the value of the game is v. Consider the game from player II's point of view.

\section{Player II LP Formulation and Solution}

Player II is trying to maximize his/her game and so it results,

Against $A_{1}: y_{1}+y_{2}+4 y_{3} \leq v$

Against $A_{2}: 4 y_{1}-2 y_{2}+4 y_{3} \leq v$

Against $A_{3}:-y_{1}+2 y_{2}-2 y_{3} \leq v$

$y_{1}+y_{2}+y_{3}=1$ [Sum of the strategies must be 1].

Substituting $\frac{y_{j}}{v}=Y_{j} ; j=1, \ldots, 3$ and then the given problem can be converted into a LP problem as follows. 
$\operatorname{Maximize} \frac{1}{v}=z=Y_{1}+Y_{2}+Y_{3}$

Subject to

$Y_{1}+Y_{2}+4 Y_{3} \leq 1$

$4 Y_{1}-2 Y_{2}+4 Y_{3} \leq 1$

$-Y_{1}+2 Y_{2}-2 Y_{3} \leq 1$

$Y_{1}, Y_{2}, Y_{3} \geq 0$

\section{Iteration 1}

Sub problem 1 Let $\lambda^{1}=0$. Then,

Maximize

$z=\left(Y_{1}+Y_{2}+Y_{3}\right)-\lambda^{1}\left(Y_{1}+Y_{2}+4 Y_{3}-1\right)$

$=\left(Y_{1}+Y_{2}+Y_{3}\right)-O\left(Y_{1}+Y_{2}+4 Y_{3}-1\right)$

$=Y_{1}+Y_{2}+Y_{3}$

subject to

$4 Y_{1}-2 Y_{2}+4 Y_{3} \leq 1,-Y_{1}+2 Y_{2}-2 Y_{3} \leq 1$

$Y_{1}, Y_{2}, Y_{3} \geq 0$

Solving by using LINDO the followings have been found, $z_{\max }=2.5, Y_{1}=0, Y_{2}=1.5, Y_{3}=1.0$

\section{Mater Problem 1}

Maximize $w=Y_{2}+Y_{3}$

subject to

$Y_{2}+4 Y_{3} \leq 1-2 Y_{2}+4 Y_{3} \leq 1$

$2 Y_{2}-2 Y_{3} \leq 1 Y_{2}, Y_{3} \geq 0$

Solving by using LINDO

$w_{\max }=0.7, Y_{2}=0.6, Y_{3}=0.1, \lambda^{2}=0.4$.

Since $z_{\max } \neq w_{\max }$, therefore next iteration has to execute.

\section{Iteration 2}

$\underline{\text { Sub problem } 2}$ Let $\lambda^{2}=0.4$. Then,

Maximize

$z=\left(Y_{1}+Y_{2}+Y_{3}\right)-0.4\left(Y_{1}+Y_{2}+4 Y_{3}-1\right)$

$=0.6 Y_{1}+0.6 Y_{2}-.6 Y_{3}+0.4$

subject to

$4 Y_{1}-2 Y+4 Y_{3} \leq 1-Y_{1}+2 Y_{2}-2 Y_{3} \leq 1$

$Y_{1}, Y_{2}, Y_{3} \geq 0$
Solving by using LINDO following results have been obtained.

$z=1.3, Y_{1}=0.66667, Y_{2}=0.833333, Y_{3}=0$

\section{$\underline{\text { Mater Problem } 2}$}

Maximize $w=Y_{1}+Y_{2}+Y_{3}$

Subject to

$Y_{1}+Y_{2}+4 Y_{3} \leq 14 Y_{1}-2 Y_{2}+4 Y_{3} \leq 1$

$-Y_{1}+2 Y_{2}-2 Y_{3} \leq 1 Y_{1}, Y_{2}, Y_{3} \geq 0$

Solving by using LINDO we get

$w_{\max }=1, Y_{1}=\frac{1}{3}, Y_{2}=\frac{2}{3}, Y_{3}=0, \lambda^{3}=1$.

Since $z_{\max } \neq w_{\max }$, therefore the next iteration has to execute.

Iteration 3

$\underline{\text { Sub problem } 3}$ Let $\lambda^{3}=1.0$. Then,

Maximize

$z=\left(Y_{1}+Y_{2}+Y_{3}\right)-1.0\left(Y_{1}+Y_{2}+4 Y_{3}-1\right)$

$=-3 Y_{3}+1$

subject to

$4 Y_{1}-2 Y+4 Y_{3} \leq 1-Y_{1}+2 Y_{2}-2 Y_{3} \leq 1$

$Y_{1}, Y_{2}, Y_{3} \geq 0$

Solving by using LINDO it is obtained.

$z_{\text {max }}=1, Y_{1}=0, Y_{2}=0.5, Y_{3}=0$.

Mater Problem 3

Maximize $w=Y_{1}+Y_{2}+Y_{3}$

subject to

$Y_{1}+Y_{2}+4 Y_{3} \leq 14 Y_{1}-2 Y_{2}+4 Y_{3} \leq 1$

$-Y_{1}+2 Y_{2}-2 Y_{3} \leq 1 Y_{1}, Y_{2}, Y_{3} \geq 0$

Solving by using LINDO the followings are found.

$w_{\max }=1, Y_{1}=\frac{1}{3}, Y_{2}=\frac{2}{3}, Y_{3}=0$.

Here it is clear that $z_{\max }=w_{\max }$, therefore optimal solution has obtained.

\section{Optimal Strategy for Player II}

Objective function value of the current LP problem is $\frac{1}{v}=w=z=1 \quad$ and the optimal solution $Y_{1}=\frac{1}{3}, Y_{2}=\frac{2}{3}, Y_{3}=0$. Therefore, the value of the game 
for the modified matrix is $\frac{1}{v}=1 \Rightarrow v=1$. Now, the value of the game by given original matrix is $v=1-k=1-0=1$, where $k=0$ (using step 3 to step 4) and mixed strategies for the original game is $y_{i}=Y_{i} v$ and so the result is $y_{1}=\frac{1}{3}, y_{2}=\frac{2}{3}, y_{3}=0$ for player II.

\section{Player I LP Formulation and Solution}

Player I is trying to minimize his/her loss in the current game, so have to substitute $\frac{x_{i}}{v}=X_{i} ; i=1, \ldots, 3$ in the original game and then the given game problem can be converted into the following LP problem.

Player I : Minimize $\frac{1}{v}=X_{1}+X_{2}+X_{3}$

subject to

$X_{1}+4 X_{2}-X_{3} \geq 1 X_{1}-2 X_{2}+2 X_{3} \geq 1$

$4 X_{1}+4 X_{2}-2 X_{3} \geq 1 X_{1}, X_{2}, X_{3} \geq 0$

\section{Iteration 1}

$\underline{\text { Sub problem } 1}$ Let $\lambda^{1}=0$.

Minimize

$z=\left(X_{1}+X_{2}+X_{3}\right)-\lambda^{1}\left(X_{1}+4 X_{2}-X_{3}-1\right)$

$=\left(X_{1}+X_{2}+X_{3}\right)-o\left(X_{1}+4 X_{2}-X_{3}-1\right)$

$=X_{1}+X_{2}+X_{3}$

subject to

$X_{1}-2 X_{2}+2 X_{3} \geq 14 X_{1}+4 X_{2}-2 X_{3} \geq 1$

$X_{1}, X_{2}, X_{\mathrm{3}} \geq 0$

Solving by using LINDO the obtained results are as follows. $z_{\text {min }}=0.7, X_{1}=0.4, X_{2}=0, X_{3}=0.3$.

\section{Master problem 1}

Minimize $w=X_{1}+X_{3}$

subject to

$\mathrm{x}_{1}+2 \mathrm{x}_{\mathrm{3}} \geq 1 X_{1}-X_{\mathrm{3}} \geq 1$

$4 X_{1}-2 X_{3} \geq 1 X_{1}, X_{3} \geq 0$

Solving by using LINDO the result is,

$w_{\text {min }}=1, X_{1}=1, X_{2}=0, X_{3}=0$ and $\lambda^{2}=1$.

Since $z_{\max } \neq w_{\max }$, then have to go to the next iteration.

\section{Iteration 2}

Sub problem 2_Let $\lambda^{1}=1$.

Minimize

$z=\left(X_{1}+X_{2}+X_{3}\right)-1.0\left(X_{1}+4 X_{2}-X_{3}-1\right)$

$=\left(X_{1}+X_{2}+X_{3}\right)-o\left(X_{1}+4 X_{2}-X_{3}-1\right)$

$=-3 X_{2}+1$

subject to

$X_{1}-2 X_{2}+2 X_{3} \geq 14 X_{1}+4 X_{2}-2 X_{3} \geq 1$

$X_{1}, X_{2}, X_{3} \geq 0$

Solving by using LINDO the obtained result is,

$z_{\text {min }}=1, X_{1}=1, X_{2}=0, X_{3}=0$.

Master problem 2

Minimize $z=X_{1}+X_{2}+X_{3}$

subject to

$X_{1}+4 X_{2}-X_{3} \geq 1 X_{1}-2 X_{2}+2 X_{3} \geq 1$

$4 X_{1}+4 X_{2}-2 X_{3} \geq 1 X_{1}, X_{2}, X_{3} \geq 0$

Solving by using LINDO the obtained result is,

$w_{\min }=1, X_{1}=1, X_{2}=0, X_{3}=0$.

Since $z_{\min }=w_{\min }$, therefore optimal solution has obtained.

\section{Optimal strategy for Player I}

Objective function value of the current LP problem is $\frac{1}{v}=w=z=1 \quad$ and the optimal solution $X_{1}=1, X_{2}=0, X_{3}=0$. Therefore, the value of the game for the modified matrix is $\frac{1}{v}=1 \Rightarrow v=1$. Now, the value of the game by given original matrix is $v=1-k=1-0=1$, where $k=0$ (using step 3 to step 4) and mixed strategies for the original game is $x_{i}=X_{i} v$ and so $x_{1}=1, x_{2}=0, x_{3}=0$ for player $\mathrm{I}$.

Decesion: Game value is $v=1$, best strategies for player I: $\left[\begin{array}{l}1 \\ 0 \\ 0\end{array}\right]$ and player II: $\left[\begin{array}{lll}\frac{1}{3} & \frac{2}{3} & 0\end{array}\right]$.

\subsection{Numerical Example 2}

Two oil companies, Bangladesh Oil Co. and Caltex, operating in a city, are trying to increase their market at the expense of the other. The Bangladesh (B. D.) Oil Co. is considering possibilities of decreasing price, giving free soft drinks on Rs. 40 purchases of oil or giving away a drinking glass with each 
40 litter purchase. Obviously, Caltex cannot ignore this and comes out with its own program to increase its share in the market. The payoff matrix forms the viewpoints of increasing or decreasing market shares is given in table below [3].

Table 1. Data for Numerical Example 2

\begin{tabular}{|l|l|l|l|}
\hline Caltex & $\begin{array}{l}\text { Decrease } \\
\text { price }\end{array}$ & $\begin{array}{l}\text { Free soft } \\
\text { drinks on } \\
\text { Rs.40 } \\
\text { purchase }\end{array}$ & $\begin{array}{l}\text { Free } \\
\text { drinking } \\
\text { glass on 40 } \\
\text { liters or so }\end{array}$ \\
\hline $\begin{array}{l}\text { Oil Co. } \\
\text { Decrease price }\end{array}$ & 4 & 1 & -3 \\
\hline $\begin{array}{l}\text { Free soft drinks } \\
\text { purchase Rs.40 }\end{array}$ & 3 & 1 & 6 \\
\hline $\begin{array}{l}\text { Free drinking } \\
\text { glass on } 40 \\
\text { liters or so }\end{array}$ & -3 & 4 & -2 \\
\hline
\end{tabular}

\section{Solution using our algorithm}

Let the value of the game is v. Consider the game from player II's point of view.

\section{Player II LP Formulation and Solution}

Player II is trying to maximize his/her game, so it have to substitute $\frac{y_{j}}{v}=Y_{j} ; j=1, \ldots, 3$ in the original game and then the given game problem can be converted into the following LP problem.

Maximize: $\frac{1}{v}=z=Y_{1}+Y_{2}+Y_{3}$

subject to

$4 Y_{1}+Y_{2}-3 Y_{3} \leq 1,3 Y_{1}+Y_{2}+6 Y_{3} \leq 1$

$-3 Y_{1}+4 Y_{2}-2 Y_{3} \leq 1, Y_{1}, Y_{2}, Y_{3} \geq 0$

\section{Iteration 1}

Sub-problem-1 Let $\lambda^{1}=0$.

Maximize $z=\left(Y_{1}+Y_{2}+Y_{3}\right)-\lambda^{1}\left(4 Y_{1}+Y_{2}-3 Y_{3}-1\right)$

$=Y_{1}+Y_{2}+Y_{3}$

subject to

$3 Y_{1}+Y_{2}+6 Y_{3} \leq 1,-3 Y_{1}+4 Y_{2}-2 Y_{3} \leq 1$

$$
Y_{1}, Y_{2}, Y_{3} \geq 0
$$

Solving by using LINDO it has found $z_{\max }=0.6, Y_{1}=$ $0.2, Y_{2}=0.4$ and $Y_{3}=0$. Here $Y_{1}, Y_{2}>0$. Therefore $Y_{3}$ will be erased from the master problem.

\section{Mater problem-1}

Maximize $w=Y_{1}+Y_{2}$

subject to

$4 Y_{1}+Y_{2} \leq 1,3 Y_{1}+Y_{2} \leq 1$

$-3 Y_{1}+4 Y_{2} \leq 1, Y_{1}, Y_{2} \geq 0$
Solving by using LINDO it has found $w_{\max }=0.526316, Y_{1}=0.157895, Y_{2}=0.368421, Y_{3}=0$ and $\lambda^{2}=0.368421$

Since $z_{\max } \neq w_{\max }$, therefore the next iteration has to execute.

\section{Iteration 2}

$\underline{\text { Sub-problem } 2} \lambda^{2}=0.368421$.

Maximize $z=\left(Y_{1}+Y_{2}+Y_{3}\right)-\lambda^{2}\left(4 Y_{1}+Y_{2}-3 Y_{3}-1\right)$ $=-0.473684 Y_{1}+0.631579 Y_{2}+2.105263 Y_{3}+$ 0.368421 subject to

$$
\begin{gathered}
3 Y_{1}+Y_{2}+6 Y_{3} \leq 1 \\
-3 Y_{1}+4 Y_{2}-2 Y_{3} \leq 1 \\
Y_{1}, Y_{2}, Y_{3} \geq 0
\end{gathered}
$$

Solving by using LINDO it has been found that $z_{\max }=$ $0.805668, Y_{1}=0, Y_{2}=0.307692$ and $Y_{3}=0.115385$. Here $Y_{1}, Y_{2}, Y_{3}>0$

\section{Master problem 2}

Maximize $z=Y_{1}+Y_{2}+Y_{3}$

subject to

$4 Y_{1}+Y_{2}-3 Y_{3} \leq 1,3 Y_{1}+Y_{2}+6 Y_{3} \leq 1$

$-3 Y_{1}+4 Y_{2}-2 Y_{3} \leq 1, Y_{1}, Y_{2}, Y_{3} \geq 0$

Solving by using LINDO it has been found that $w_{\max }=$ $0.571429, Y_{1}=0.167702, Y_{2}=0.385093, Y_{3}=0.0186335$ and $\lambda^{3}=0.142857$

Since $z_{\max } \neq w_{\max }$, therefore the next iteration has to execute.

\section{Iteration 3}

$\underline{\text { Sub problem } 3} \lambda^{3}=0.142857$

Maximize $z=\left(Y_{1}+Y_{2}+Y_{3}\right)-\lambda^{3}\left(4 Y_{1}+Y_{2}-3 Y_{3}-1\right)$

$=\left(Y_{1}+Y_{2}+Y_{3}\right)-0.142857\left(4 Y_{1}+Y_{2}-3 Y_{3}-1\right)$

$=0.428572 Y_{1}+0.857143 Y_{2}+1.428571 Y_{3}+0.142857$

subject to

$3 Y_{1}+Y_{2}+6 Y_{3} \leq 1,-3 Y_{1}+4 Y_{2}-2 Y_{3} \leq 1$

$$
Y_{1}, Y_{2}, Y_{3} \geq 0
$$

Solving by using LINDO it has been found that $z_{\max }=$ $0.571429, Y_{1}=0, Y_{2}=0.307692$ and $Y_{3}=0.115385$. Here $Y_{1}, Y_{2}, Y_{3}>0$.

\section{Master problem 3}

Maximize $z=Y_{1}+Y_{2}+Y_{3}$ subject to

$4 Y_{1}+Y_{2}-3 Y_{3} \leq 1,3 Y_{1}+Y_{2}+6 Y_{3} \leq 1$

$-3 Y_{1}+4 Y_{2}-2 Y_{3} \leq 1, Y_{1}, Y_{2}, Y_{3} \geq 0$

Solving by using LINDO is has been found that $w_{\max }=$ $0.571429, Y_{1}=0.167702, Y_{2}=0.385093, Y_{3}=0.0186335$. Since $z_{\max }=w_{\max }$ therefore optimal solution has obtained.

\section{Optimal Strategy for Player II}

Objective function value of the current LP is $\frac{1}{v}=w=z=0.571429$ and optimal solution is 
$Y_{1}=0.571429, Y_{2}=0.385093, Y_{3}=0.0186335$.

Therefore, the value of the game for the modified matrix is $\frac{1}{-}=0.571429 \Rightarrow v=1.75$. Now, the value of the game by given original matrix is $v=1.75-k=1.75-0=1.75$, where $k=0$ (using step 3 to step 4) and mixed strategies for the original game is $y_{i}=Y_{i} v$ and so the followings are found.

$y_{1}=1.75 * 0.167702=0.293782$,

$y_{2}=1.75 * 0.38509=0.673912$,

$y_{3}=1.75 * 0.018633=0.032608$ for player II.

\section{Player II LP Formulation and Solution}

Again, Player I is trying to minimize his/her loss in the current game, so it have to substitute $\frac{x_{i}}{v}=X_{i} ; i=1, \ldots, 3$ in the original game and then the given game problem can be converted into the following LP problem.

Player I : Minimize $\frac{1}{v}=X_{1}+X_{2}+X_{3}$

subject to

$4 X_{1}+3 X_{2}-3 X_{3} \geq 1, X_{1}+X_{2}+4 X_{3} \geq 1$

$-3 X_{1}+6 X_{2}-2 X_{3} \geq 1, X_{1}, X_{2}, X_{3} \geq 0$

\section{Iteration 1}

Sub problem 1 Let $\lambda^{1}=0$.

Minimize $\quad z=\left(X_{1}+X_{2}+X_{3}\right)-\lambda^{1}\left(4 X_{1}+3 X_{2}-3 X_{3}-\right.$ 1) $=X_{1}+X_{2}+X_{3}$ subject to

$X_{1}+X_{2}+4 X_{3} \geq 1,-3 X_{1}+6 X_{2}-2 X_{3} \geq 1$

$$
X_{1}, X_{2}, X_{3} \geq 0
$$

Solving by using LINDO it has been found that $z_{\text {min }}=$ $0.423077, X_{1}=0, X_{2}=0.230769, X_{3}=0.192308$. Here $X_{1}, X_{2}, X_{3}>0$.

\section{Master problem 1}

Minimize $w=X_{2}+X_{3}$

subject to

$3 X_{2}-3 X_{3} \geq 1, X_{2}+4 X_{3} \geq 1$

$6 X_{2}-2 X_{3} \geq 1, X_{2}, X_{3} \geq 0$

Solving by using LINDO it has been found that $w_{\text {min }}=$ $0.6, X_{1}=0, X_{2}=0.466667, X_{3}=0.133333$ and $\lambda^{2}=0.2$.

Since $z_{\text {max }} \neq w_{\max }$, therefore the next iteration has to execute.

\section{Iteration 2}

Sub problem $2 \lambda^{2}=0.2$.

Minimize $\quad z=\left(X_{1}+X_{2}+X_{3}\right)-\lambda^{2}\left(4 X_{1}+3 X_{2}-3 X_{3}-\right.$ 1) $=\left(X_{1}+X_{2}+X_{3}\right)-0.2\left(4 X_{1}+3 X_{2}-3 X_{3}-1\right)$

$=0.2 X_{1}+0.4 X_{2}+1.6 X_{3}+0.2$ subject to

$X_{1}+X_{2}+4 X_{3} \geq 1,-3 X_{1}+6 X_{2}-2 X_{3} \geq 1$

$$
X_{1}, X_{2}, X_{3} \geq 0
$$

Solving by using LINDO it has been found that $z_{\text {min }}=$ $0.488889, X_{1}=0.555556, X_{2}=0.444444, X_{3}=0$.

\section{Master problem 2}

Minimize $w=X_{1}+X_{2}+X_{3}$

subject to

$$
\begin{gathered}
4 X_{1}+3 X_{2}-3 X_{3} \geq 1 \\
X_{1}+X_{2}+4 X_{3} \geq 1 \\
-3 X_{1}+6 X_{2}-2 X_{3} \geq 1 \\
X_{1}, X_{2}, X_{3} \geq 0
\end{gathered}
$$

Solving by using LINDO it has been found that $w_{\min }=$ $0.571429, X_{1}=0.142857, X_{2}=0.285714, X_{3}=0.142857$ and $\lambda^{3}=0.167702$

Since $z_{\max } \neq w_{\max }$, therefore to the next iteration has to execute.

\section{Iteration 3}

$\underline{\text { Sub problem } 3} \lambda^{3}=0.167702$.

Minimize $\quad z=\left(X_{1}+X_{2}+X_{3}\right)-\lambda^{3}\left(4 X_{1}+3 X_{2}-3 X_{3}\right.$ 1) $=\left(X_{1}+X_{2}+X_{3}\right)-0.167702\left(4 X_{1}+3 X_{2}-3 X_{3}-1\right)=$ $0.329192 X_{1}+0.496894 X_{2}+1.503106 X_{3}+$ 0.167702 subject to

$$
\begin{gathered}
X_{1}+X_{2}+4 X_{3} \geq 1 \\
-3 X_{1}+6 X_{2}-2 X_{3} \geq 1 \\
X_{1}, X_{2}, X_{3} \geq 0
\end{gathered}
$$

Solving by using LINDO it has been found that $z_{\text {min }}=$ $0.571429, X_{1}=0.555556, X_{2}=0.444444, X_{3}=0$.

\section{$\underline{\text { Master problem } 3}$}

Minimize $w=X_{1}+X_{2}+X_{3}$

subject to

$4 X_{1}+3 X_{2}-3 X_{3} \geq 1, X_{1}+X_{2}+4 X_{3} \geq 1$

$-3 X_{1}+6 X_{2}-2 X_{3} \geq 1, X_{1}, X_{2}, X_{3} \geq 0$

Solving by using LINDO it has been found that $w_{\min }=$ $0.571429, X_{1}=0.142857, X_{2}=0.285714, X_{3}=0.142857$.

Since $z_{\max }=w_{\max }$ therefore optimal solution has obtained.

\section{Optimal Strategy for Player I}

Objective function value of the current LP is $\frac{1}{v}=w=z=0.571429 \quad$ and optimal solution $X_{1}=0.142857, X_{2}=0.285714, X_{3}=0.142857$ .Therefore, the value of the game for the modified matrix is $\frac{1}{v}=0.571429 \Rightarrow v=1.75$. Now, the value of the game by given original matrix is $v=1.75-k=1.75-0=1.75$, where $k=0$ (using step 3 to step 4) and mixed strategies for the original game is 
$y_{i}=Y_{i} v$ and so the obtained result is $x_{1}=0.25, x_{2}=0.45, x_{3}=0.25$ for player $\mathrm{I}$.

Decesion: Game value is $v=1.75$, best strategies for player I: $\left[\begin{array}{c}0.25 \\ 0.50 \\ 0.25\end{array}\right]$ and player II:

$\left[\begin{array}{lll}0.293782 & 0.673912 & 0.032608\end{array}\right]$.

\subsection{Numerical Example 3}

The payoff matrix of a game is given below.

Table.2: Data for Numerical Example 3

\begin{tabular}{|c|c|c|c|c|c|c|}
\hline A $_{\mathrm{B}}$ & I & II & III & IV & V & VI \\
\hline 1 & 4 & 2 & 0 & 2 & 1 & 1 \\
\hline 2 & 4 & 3 & 1 & 3 & 2 & 2 \\
\hline 3 & 4 & 3 & 7 & -5 & 1 & 2 \\
\hline 4 & 4 & 3 & 4 & -1 & 2 & 2 \\
\hline 5 & 4 & 3 & 3 & -2 & 2 & 2 \\
\hline
\end{tabular}

Find the best strategy for each player, and the value of the game to $\mathrm{A}$ and $\mathrm{B}[3]$.

\section{Exact Solution}

The Solution can be found step by step in [3].

The value of the game is $13 / 7$. The strategy for player $\mathrm{A}$ is $[0$, $6 / 7,1 / 7,0,0]$ and strategy for player $\mathrm{B}$ is $[0,0,4 / 7,3 / 7,0,0]$.

\section{Player II's LP Formulation}

Let the value of the game is v. Consider the game from player II's point of view. Player II is trying to maximize his/her game and so the followings have been found,

$$
\begin{aligned}
& 4 y_{1}+2 y_{2}+2 y_{4}+y_{5}+y_{6} \leq v \\
& 4 y_{1}+3 y_{2}+y_{3}+3 y_{4}+2 y_{5}+2 y_{6} \leq v \\
& 4 y_{1}+3 y_{2}+y_{3}-5 y_{4}+y_{5}+2 y_{6} \leq v \\
& 4 y_{1}+3 y_{2}+4 y_{3}-y_{4}+2 y_{5}+2 y_{6} \leq v \\
& 4 y_{1}+3 y_{2}+3 y_{3}-2 y_{4}+2 y_{5}+2 y_{6} \leq v
\end{aligned}
$$

Now, it has to substitute $\frac{y_{j}}{v}=Y_{j} ; j=1, \ldots, 6$ in the original game and then the given game problem can be converted into the following LP problem.

Maximize : $\frac{1}{v}=Y_{1}+Y_{2}+Y_{3}+Y_{4}+Y_{5}+Y_{6}$ subject to

$$
\begin{aligned}
& 4 Y_{1}+2 Y_{2}+2 Y_{4}+Y_{5}+Y_{6} \leq 1 \\
& 4 Y_{1}+3 Y_{2}+Y_{3}+3 Y_{4}+2 Y_{5}+2 Y_{6} \leq 1 \\
& 4 Y_{1}+3 Y_{2}+Y_{3}-5 Y_{4}+Y_{5}+2 Y_{6} \leq 1 \\
& 4 Y_{1}+3 Y_{2}+4 Y_{3}-Y_{4}+2 Y_{5}+2 Y_{6} \leq 1 \\
& 4 Y_{1}+3 Y_{2}+3 Y_{3}-2 Y_{4}+2 Y_{5}+2 Y_{6} \leq 1 \\
& Y_{j} \geq 0 ; j=1, \ldots, 6
\end{aligned}
$$

\section{Player I's LP Formulation}

Now have to consider the game from player I's point of view. Player I is trying to minimize his/her game and so,

$$
\begin{aligned}
& 4 x_{1}+4 x_{2}+4 x_{3}+4 x_{4}+4 x_{5} \geq v \\
& 2 x_{1}+3 x_{2}+3 x_{3}+3 x_{4}+3 x_{5} \geq v \\
& 0 x_{1}+x_{2}+7 x_{3}+4 x_{4}+3 x_{5} \geq v \\
& 2 x_{1}+3 x_{2}-5 x_{3}-x_{4}-2 x_{5} \geq v \\
& x_{1}+2 x_{2}+x_{3}+2 x_{4}+2 x_{5} \geq v \\
& x_{1}+2 x_{2}+2 x_{3}+2 x_{4}+2 x_{5} \geq v
\end{aligned}
$$

substitute $\frac{x_{i}}{v}=X_{i} ; i=1, \ldots, 5$ in the above LP then the followings are obtained,

Minimize: $\frac{1}{v}=X_{1}+X_{2}+X_{3}+X_{4}+X_{5}$

subject to

$$
\begin{aligned}
& 4 X_{1}+4 X_{2}+4 X_{3}+4 X_{4}+4 X_{5} \geq 1 \\
& 2 X_{1}+3 X_{2}+3 X_{3}+3 X_{4}+3 X_{5} \geq 1 \\
& 0 . X_{1}+X_{2}+7 X_{3}+4 X_{4}+3 X_{5} \geq 1 \\
& 2 X_{1}+3 X_{2}-5 X_{3}-X_{4}-2 X_{5} \geq 1 \\
& X_{1}+2 X_{2}+X_{3}+2 X_{4}+2 X_{5} \geq 1 \\
& X_{1}+2 X_{2}+2 X_{3}+2 X_{4}+2 X_{5} \geq 1 \\
& X_{i} \geq 0 ; i=1, \ldots, 5
\end{aligned}
$$

This example is a $5 \times 5$ payoff matrix and it is very difficult to solve manually. That's why in the Section 5, Computer codes have been developed by using AMPL according to the introduced algorithms. The code consists of three different parts. These are AMPL model file, data file and run file.

\section{COMPUTER ALGEBRA}

In this Section, a general computer technique has been developed by using AMPL by considering algorithms 
prescribed in Section 3 which can be worked for two person zero sum game problems. Every AMPL program is composed of three portions as model file, run file and data file. Model file contains the necessary parameter and variables declaration and the basic formulation. Data file and run files contain the inputs and formatting output commands respectively. Due to volume of the paper, computer codes are not given here.

\subsection{AMPL Output Part I}

In the current Section, the AMPL outputs have been presented of the numerical examples prescribed in Section 4 by considering the algorithm in Section 3 and the computer algebra.

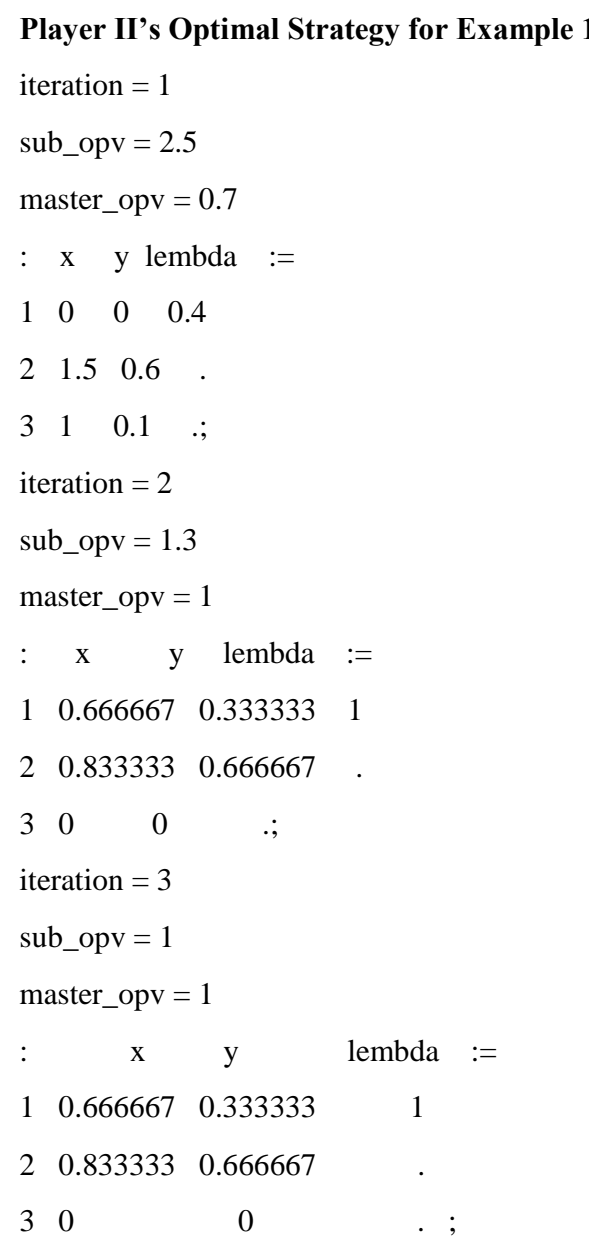

\section{Discussions}

From the above AMPL output for numerical example 1, it observes that sub-problem and master-problem value become equal at the third iteration. From computer code obtained decision variable values are $1 / 3,2 / 3$, and 0 respectively and objective function value is 1 for Player II's LP problem. Therefore, the value of the game by given original matrix is $v=\frac{1}{1}$ and mixed strategies for the original game is $y_{1}=\frac{1}{3}, y_{2}=\frac{2}{3}, y_{3}=0$ for player II.

\section{Player I's Optimal Strategy for Example 1}

iteration $=1$

sub_opv $=-0.7$

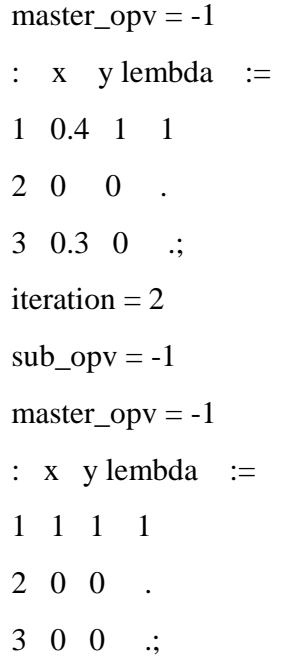

Here one has to modify our code to solve a minimization problem. We converted the minimization problem into maximization problem by multiplying with minus one.

\section{Discussion}

From the AMPL output obtained optimal decision variable values are 1,0 , and 0 . Objective function value $=-(-1)=1$. Therefore, optimal solution for Player I's LP is $[1,0,0]$ and optimal value of the LP is 1 . Hence, the value of the game by given original matrix is $v=\frac{1}{1}$ and mixed strategies for the original game is $x_{1}=1, x_{2}=0, x_{3}=0$ for player $\mathrm{I}$

\section{Player II's Optimal Strategy for Example 2}

iteration $=1$

sub_opv $=0.6$

master_opv $=0.526316$

$\begin{array}{llcc}: & \mathrm{x} & \mathrm{y} & \text { lembda }:= \\ 1 & 0.2 & 0.157895 & 0.368421 \\ 2 & 0.4 & 0.368421 & . \\ 3 & 0 & 0 & . ; \\ \text { iteration }=2 \\ \text { sub_opv }=0.805668 \\ \text { master_opv }=0.571429\end{array}$

$\begin{array}{lccc}: & \mathrm{x} & \mathrm{y} & \text { lembda }:= \\ 1 & 0 & 0.167702 & 0.142857 \\ 2 & 0.307692 & 0.385093 & . \\ 3 & 0.115385 & 0.0186335 & . ; \\ \text { iteration }=3 & & \\ \text { sub_opv }=0.571429 & \\ \text { master_opv }=0.571429 & \\ : & \mathrm{x} & \mathrm{y} & \text { lembda } \quad:= \\ 1 & 0 & 0.167702 & 0.142857 \\ 2 & 0.307692 & 0.385093 & . \\ 3 & 0.115385 & 0.0186335 & . \quad ;\end{array}$




\section{Discussion}

From AMPL output of numerical example 2 optimal solution has been obtained at third iteration. Here optimal decision variable values are 0.167702 ,

0.385093 and 0.0186335 . Game value is 0.571429 . Therefore, the value of the game by given original matrix is $v=\frac{1}{0.571429}=1.75$ and mixed strategies for the original game is $y_{1}=0.293782, y_{2}=0.673912$, $y_{3}=0.032608$ for player II.

\section{Player I's Optimal Strategy for Example 2}

iteration $=1$

sub_opv $=-0.423077$

master_opv $=-0.6$

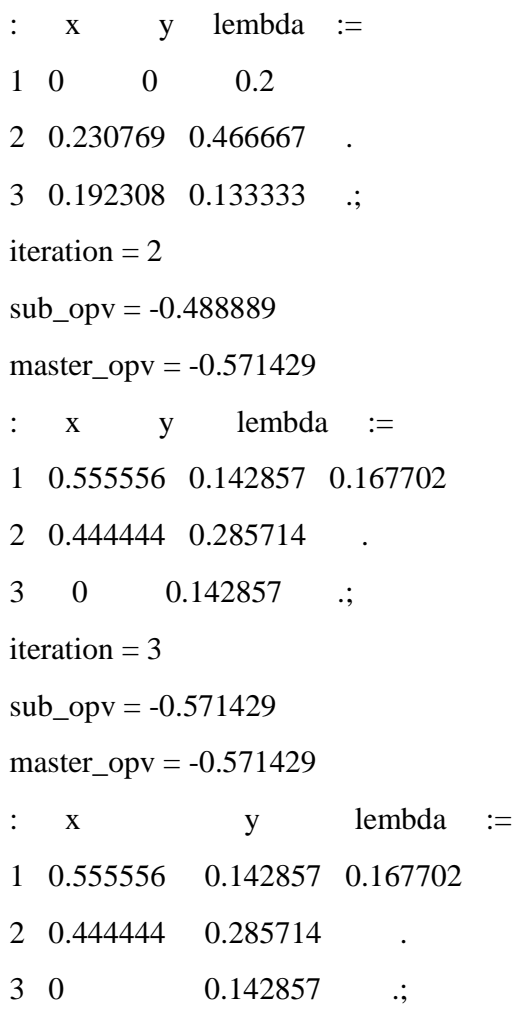

\section{Discussion}

From the AMPL output of numerical example 2 it has been observed that optima decision variable values are 0.142857 , 0.285714 and 0.142857. Game value $=-(-0.571429)=$ 0.571429 .

Therefore, optimal strategy for Player I is [0.142857, $0.285714,0.142857]$ and for Player II is [0.167702,0.385093, $0.0186335]$. Game value is 0.571429 . Therefore, the value of the game by given original matrix is $v=\frac{1}{0.571429}=1.75$ and mixed strategies for Player II is:

$x_{1}=0.25, x_{2}=0.45, x_{3}=0.25 y_{1}=0.293782$.

\section{Player II's Optimal Strategy for Example 3}

iteration $=1$

sub_opv $=10.25$

master_opv $=0.25$

: $\mathrm{x}$ y lembda :=

$\begin{array}{llll}1 & 0 & 0 & 0\end{array}$

200.

$\begin{array}{lll}3 & 0.25 & 0.25\end{array}$

$\begin{array}{lll}4 & 0 & 0\end{array}$

$5 \quad 0 \quad 0$

$\begin{array}{llll}6 & 0 & 0 & ;\end{array}$

iteration $=2$

sub_opv $=0.538462$

master_opv $=0.538462$

$\begin{array}{lllc}: & \mathrm{x} & \mathrm{y} & \text { lembda } \\ 1 & 0 & 0 & 0 \\ 2 & 0 & 0 & . \\ 3 & 0.307692 & 0.307692 & . \\ 4 & 0.230769 & 0.230769 & . \\ 5 & 0 & 0 & . \\ 6 & 0 & 0 & .\end{array}$

\section{Discussion}

From AMPL out we observe that optimal solution has been obtained at second iteration. Optimal decision variable values are $0,0,0.307692,0.230769,0$ and 0 . Optimal value is 0.538462 for the LP problem. So the game value is $\frac{1}{0.538462}=1.857$ and opimal strategy of the given game problem is $[0,0,0.57,0.43,0,0]$ for Player II.

The value of the game is $13 / 7$. The strategy for player $\mathrm{A}$ is [0, $6 / 7,1 / 7,0,0]$ and strategy for player B is $[0,0,4 / 7,3 / 7,0,0]$.

\section{Player I's Optimal Strategy for Example 3}

iteration $=1$

sub_opv $=11$

master_opv $=-0.538462$

$\begin{array}{llccc}: & x & y & \text { lembda } & := \\ 1 & 0 & 0 & 0 \\ 2 & 0.461538 & 0.461538 \\ 3 & 0.0769231 & 0.0769231 \\ 4 & 0 & 0 & \cdot \\ 5 & 0 & 0 & . \\ \text { iteration }=2 & \\ \text { sub_opv }= & -0.538462 \\ \text { master_opv }= & -0.538462\end{array}$

: $\mathrm{x} \quad \mathrm{y}$ lembda := 


$\begin{array}{llcc}1 & 0 & 0 & 0 \\ 2 & 0.461538 & 0.461538 & . \\ 3 & 0.0769231 & 0.0769231 & . \\ 4 & 0 & 0 & . \\ 5 & 0 & 0 & \text {.; }\end{array}$

\section{Discussion}

We obtain optimal solution for Player I's LP at second iteration. Optimal decision variable values are $0,0.461538$, $0.0769231,0$ and 0 for the Player I's LP and functional value $=-(-0.538462)=0.538462$.

Therefore, the game value for player $I$ is $\frac{1}{0.538462}=1.857$ and the optimal strategy for Player I is $[0,0.8571,0.1428,0,0]$.

\section{CONVERGENCE COMPARISON}

To obtain the graphical representation of the convergence of master and sub problem values, few commands of MATHEMATICA has been used [7] by considering the algorithm prescribed in Section 3.

\section{Master-Sub Problem value for Example 1}

The convergence of the Sub-problem and Master-problem values for Player II has been presented by the following figure.

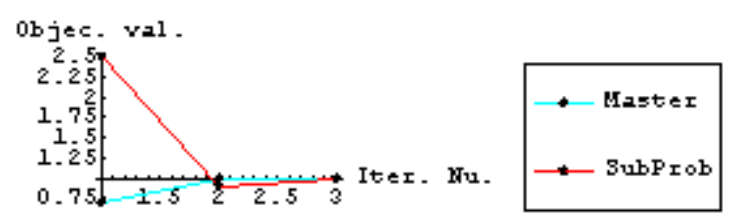

Fig.1: Convergence for Player II

Again the convergence of the Sub-problem and Masterproblem values for Player II has been presented by the following figure.

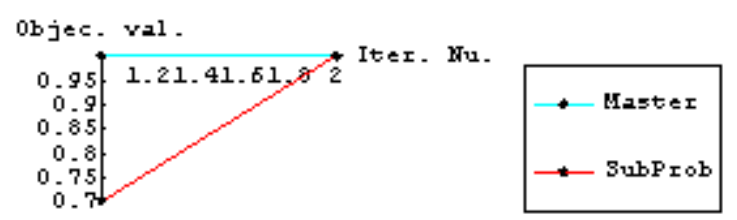

Fig.2: Convergence for Player I

Master-Sub Problem value for Problem 2

The convergence of the Sub-problem and Master-problem values for Player II has been presented by the following figure.

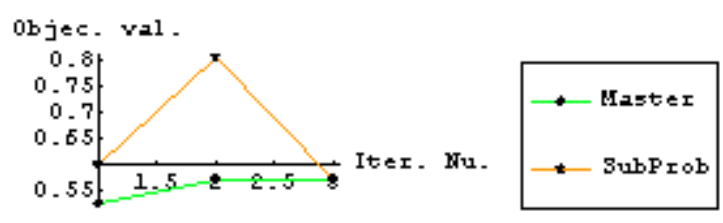

Fig.3: Convergence for Player II

The convergence of the Sub-problem and Master-problem values for Player II has been presented by the following figure.

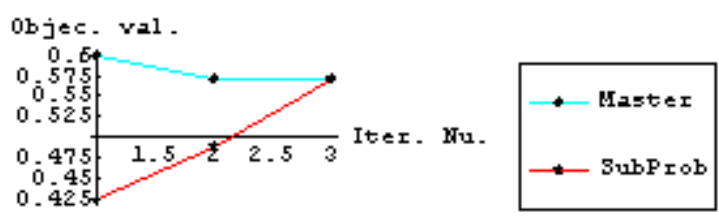

Fig.4: Convergence for Player I

Remark: The dual values taken in each case of the first iteration was a random choice. If one took the value of the dual variable as exactly then have in the iteration which gives optimal value, then would meet the optimal condition after a single iteration.

\section{CONCLUSION}

In this paper, a new decomposition based algorithm with computer code had presented for analyzing two person zero sum game problems. It was solved by using the recent developed AMPL code. An analysis about the decomposition procedure had shown. It also showed that the ability of our program in saving labor and time for solving game problems by analyzing a number of numerical examples. In future, we would concentrate on applying the Dantzig-Wolfe decomposition method of two person zero sum game problems and try to find other kinds of decomposition methods by considering suitable technique.

\section{REFERENCES}

[1] Davis M., 1983. Game Theory: An Introduction, Basic Books, Ney York.

[2] Dantzig, G.B. and P. Wolfe, 1961. The Decomposition Algorithm for Linear Programming, Econometrica, 29 (4).

[3] Das H. K., T. Saha and M. Babul Hasan, 2012. Numerical Experiments By Improving a Numerical Methods For Solving Game Problems Through Computer Algebra, International Journal of Decision Sciences, 3(1), 23-52.

[4] Das H.K. and M. Babul Hasan, 2011. An Algorithm and Its Computer Technique for Solving Game Problems Using LP method, Int. J. of Basic \& Applied Sciences", 11(3), 90-99.

[5] Dantzig, G.B., 1963. Linear Programming and Extensions, Princeton University Press, Princeton, U.S.A. 
[6] Fourer, R., D.M. Gay and B.W. Kernighan, 2003. A Modeling Language for Mathematical Programming, Second edition, Thomson Publication.

[7] Winston, W.L., 1994. Linear Programming: Applications and Algorithm, Duxbury press, Belmont, California, U.S.A.

[8] Eugene Don. Theory and Problems of Mathmatica, McGRAW-HILL, Schaum's Outline Series, New York, San Francisco Washington, D.C.

[9] Nisan N., Roughgarden T., Tardos E., Vazirari V.V., “ Algorithmic Game Theory", Cambridge University Press, 2007.

[10] Von Neumann J., and O. Morgenstern, "Theory of Games and Economic Behavior", second edition, Princeton University Press, Princeton, N.J., 1947.

[11] Von Neumann J., 1928. Zur Theorie der Gesselschaftsspiele, Mathematische Annalen 100.

[12] Mamer, J. W. \& R. D. McBride, 2000. A Decomposition-based Pricing Procedure for Large- Scale Linear Programs: An application to the linear multicommodity Flow Problem, 46(5), 693-709.
[13] Dantzig, G. B., \& P. Wolfe, 1961. The Decomposition Algorithm for Linear Programming, Econometrica, 29(4), 767-778

[14] Libbecke, M. E. \& J. Desrosiers, 2005. Selected topics in column generation, Operations research, 53(6), 10071023 .

[15] Das H.K. and M. Babul Hasan, 2011. An Algorithm and Its Computer Technique for Solving Game Problems Using LP method, Int. J. of Basic \& Applied Sciences", 11(3), 90-99.

[16] Arshaful Islam, M. Babul Hasan and H.K. Das, 2014. A new Decomposition-Based Pricing Technique for Solving Large-Scale Mixed IP with a Computer Technique, GANIT, Bangladesh Mathematical Society, 34, 5-20

[17] H. K. Das and M. Babul Hasan, 2013. An Improved Decomposition Approach and Its Computer Technique for Solving Primal Dual LP \& LFP Problems, GANIT, J. Bangladesh Math. Society, 33, 65-75. 